\title{
Brian Stimpson, Paul Valéry, L'écriture en devenir, Frankfurt am Main, Éditions Peter Lang, 2009, 432
} p.

Jean-Michel Maulpoix

\section{(2) OpenEdition} Journals

Édition électronique

URL: http://journals.openedition.org/genesis/648

DOI : $10.4000 /$ genesis. 648

ISSN : 2268-1590

Éditeur :

Presses universitaires de Paris Sorbonne (PUPS), Société internationale de génétique artistique littéraire et scientifique (SIGALES)

\section{Édition imprimée}

Date de publication : 30 octobre 2011

Pagination : 189-190

ISBN : 978-2-84050-804-5

ISSN : $1167-5101$

\section{Référence électronique}

Jean-Michel Maulpoix, «Brian Stimpson, Paul Valéry, L'écriture en devenir, Frankfurt am Main, Éditions Peter Lang, 2009, 432 p. », Genesis [En ligne], 33 | 2011, mis en ligne le 23 octobre 2013, consulté le 22 septembre 2020. URL : http://journals.openedition.org/genesis/648 ; DOI : https://doi.org/10.4000/ genesis. 648 
développement supplémentaire. Genèses théâtrales constitue en somme un jalon majeur dans une enquête sur la création théâtrale que, loin de clore, il relance avec une ambition et une force inédites.

Hubert Thüring, Corinna Jäger-Trees, Michael Schläfli (dir.), Anfangen zu schreiben. Ein kardinales Moment von Textgenese und Schreibprozess im literarischen Archiv des 20. Jahrhunderts [Commencer à écrire/Une étape essentielle dans la genèse du texte et le processus d'écriture, d'après les archives littéraires du $\mathbf{x x}^{\mathrm{e}}$ siècle], München, Fink Verlag, 2009, 345 p.

\section{Compte rendu par Louis Hay}

On ne sait pas toujours en France que la Suisse aussi est terre de génétique. En Romandie, les chercheurs travaillent de longue date sur les archives littéraires fondées à Lausanne autour du fonds Ramuz. À Berne, le «Schweizerisches Literaturarchiv », créé à l'initiative de Dürrenmatt, a lancé un programme intitulé « Textgenese und Schreibprozess » auquel revient l'initiative du colloque de 2007 dont rend compte le présent volume de la collection "Zur Genealogie des Schreibens ». La rencontre porte sur une problématique classique de la critique génétique, celle des lieux stratégiques de l'écriture, un sujet qui a d'ailleurs été discuté dans plusieurs séminaires de l'ITEM 1 . C'est le problème des débuts qui est à l'ordre du jour ici et qui induit une grande variété d'approches.

Plusieurs études visent une typologie des débuts, qui s'étend du programmatique au processuel. Particulièrement intéressante est à cet égard la contribution de Corinna Jäger-Trees consacrée aux manuscrits du romancier suisse Otto F. Walter : l'écrivain y passe d'une écriture programmée (Der Stumme, 1959) à une rédaction immédiate (Zeit des Fasans, 1988). Notre habitude d'attribuer une écriture caractéristique à tout écrivain mérite peut-être réflexion.
Bernhild Boie analyse trois « entrées en écriture » parfaitement dissemblables (Heym, Eich, Rühmkorf). Écartant toute ambition typologique elle conclut, à l'instar de Gracq : «il n’y a que des cas d'espèce ». Cette position se trouve nuancée ailleurs : Hubert Thüring affirme que tout écrivain pense à la réception de son œuvre lorsqu'i commence à écrire, Stephan Kammer voit dans l'écriture une " technique culturelle complexe » et non une simple entreprise individuelle.

D'autres essais portent sur les fonctions esthétiques des commencements. Dans un manuscrit de Paul Celan, Sandro Zanetti souligne la fonction du titre, apparu à la fin de la genèse mais placé au début du texte pour en rappeler le statut initial : un « Projet», une « Ébauche ». Chez Robert Walser, Wolfram Groddeck trouve dans le texte même une contrainte qui s'impose au début : l'impossibilité d'annoncer une construction logique pour le livre à venir. Et dans les Notices d'Elias, Irmgard Wirtz retrouve les raisons qui rendent impossible l'existence même d'un début «Ce qui doit ici recevoir une durée, c'est le "Texte-Vie" qui ne doit comporter nul début, car la fin alors deviendrait visible », écrit l'auteur.

En changeant de thématique, un dernier groupe de contributions s'intéresse au commencement de l'écriture en tant que sujet littéraire. Hubert Thüring observe, dans les romans de Glauser, une « spirale » qui relie le mouvement de l'écriture à des éléments d'autoreprésentation. Dans les débuts de Kafka, Alexander Honold relève une réflexion sur l'acte d'écrire qui accompagne constamment le mouvement de la plume. Andreas Maunz enfin, s'intéresse aux écrits de Vassula Ryden, une mystique de l'Église grecque orthodoxe. Dans son recueil de messages True Life in God (1986-2003) il n'est, pour l'écrit, ni de début ni de fin puisque tout message de Dieu manifeste sa propre complétude. La tentative d'une " poétologie de l'écriture sacrée » se trouve cependant bornée par l'horizon du texte ; les manuscrits demeurent hors de portée.
Dans leur ensemble, les contributions du volume sont d'excellente qualité, même si les plus intéressantes se trouvent parmi les études de corpus les plus poussées. Le livre se recommande à tous les généticiens germanophones : il montre parfaitement tout ce qui reste à faire sur l'un des sujets les mieux étudiés de la génétique.

Brian Stimpson, Paul Valéry. L'écriture en devenir, Frankfurt am Main, Peter Lang, 2009, 432 p.

\section{Compte rendu par Jean-Michel} Maulpoix

Davantage que ses œuvres, si accomplies soient-elles, il se pourrait que ce soit le travail d'un écrivain qui le garde vivant, longtemps après sa disparition. Ainsi ne donne-t-il pas seulement à lire des textes, mais à interroger encore un geste de plume qui paraît se poursuivre sous nos yeux. Il en va ainsi pour Paul Valéry, tel que Brian Stimpson nous invite à le redécouvrir en rouvrant dans un volumineux essai intitulé L'Écriture en devenir le chantier de La Jeune Parque et de « La Pythie », à la lumière de documents manuscrits inédits parmi lesquels des lettres que Valéry a adressées à sa femme entre 1908 et 1918.

Brian Stimpson a suivi d'une façon très attentive (l'on est tenté d'écrire « très attentionnée ») Paul Valéry au travail, en cheminant à travers les brouillons de ses poèmes et sa correspondance, en jetant un double regard sur la poussée génétique manifeste dans les manuscrits qui permet d'appréhender l'évolution de l'écriture, et sur le processus de composition qui conduit à interroger plus globalement les moyens et la pratique de l'écrivain. Ainsi éclaire-t-il non seulement les textes, mais également la

1. Voir notamment Bernhild Boie et Daniel Ferrer (dir.), Genèses du roman contemporain. Incipit et entrée en écriture, Paris, CNRS Éditions, 2e éd., 1993 ; Claude Duchet et Isabelle Tournier (dir.), Genèses des fins, SaintDenis, PUV, 1996. 
sensibilité artistique de Valéry avant 1912 et se trouve-t-il conduit à accorder une place décisive à « la musique intime du moi », étrange énergie jusqu'ici peu observée semble-t-il dans les études valéryennes.

C'est alors un passionnant portrait d'écrivain à l'ouvrage qui se dessine, largement « autoanalysé » par Valéry lui-même, où sensibilité et intelligence sont aux prises et forment un thyrse complexe. Brian Stimpson met en lumière les rythmes de la création valéryenne où se succèdent un " temps de latence créatrice » durant lequel « l'ignorance même est dynamique », et un temps de " découverte réfléchie » où la question $\mathrm{du}$ 《faire » se trouve posée à la fois sous l'angle du quoi et du comment. Il apparaît que l'essentiel du travail consiste en rapprochements et en combinaisons. L'écriture de Valéry présente un caractère singulièrement évolutif et tâtonnant. Brian Stimpson montre comment le poème sort de sa gangue, se dégage de ses ratures, la manière dont la pensée se clarifie : le travail de la langue est travail de l'esprit.

$\mathrm{Au}$ commencement est un potentiel dont il faudra débrouiller une forme, des possibles d'où extraire des nécessités, une matière germinale pareille à quelque « soupe primordiale ». Paul Valéry, à maints égards, est un explorateur et, avant la lettre, son propre généticien : il ne quitte pas des yeux le mûrissement de son travail et en développe l'analyse. N'est-ce pas là, après tout, ce qui dans l'écriture l'intéresse le plus : y voir l'esprit fonctionner ? Comme l'observe Brian Stimpson, « l'écrire des Cahiers et la pratique des manuscrits de la poésie sont intimement imbriqués ».

Pour emboîter le pas sans faillir à un pareil explorateur, il fallait un soin et un souffle de spéléologue : c'est avec autant de prudence que de qualité d'attention et de méticulosité critique que Brian Stimpson offre ces nouvelles entrées dans le temps de l'écriture et dans le faire du poète, ce faire vorace qui lui-même interroge, brasse et assimile les matériaux les plus divers. Il a su ne pas figer Valéry à partir du texte connu et maintes fois déjà commenté ; il est revenu aux sources au lieu de procéder à rebours et d'effacer du même coup ces hésitations et ces glissements imperceptibles qui sont la vie même de l'écrit.

Ainsi ce livre aussi riche que sérieux autorise-t-il de troublantes retrouvailles. Émouvante en effet est la réalité prise par la voix de Valéry dans cette étude : par le poème, la pensée et les détours complexes de l'écrit, mais aussi par des lettres intimes (telles celles écrites à sa femme depuis Perros-Guirec en juillet 1913)... Le tout confère à Valéry une présence rare dans un livre de cette espèce. Valéry au travail, ciselant le son et le sens, et Valéry sensible, sensoriel, frissonnant parfois, n'y font qu'un.

Le Signe et la consigne. Essai sur la genèse de l'ouvre en régime naturaliste. Zola, dir. Philippe Hamon, Genève, Droz, 2009, 320 p.

\section{Compte rendu par Céline Grenaud}

« Nos œuvres en gestation échappent absolument à notre volonté », écrivait Zola à Huysmans, dans une lettre du 20 mai 1884. De fait, la question se pose de savoir si la création coïncide avec la mise en œuvre de processus parfaitement maîtrisés, facilement repérables et systématiquement appliqués ou bien si elle échappe à la conscience et reste placée, en définitive, sous le signe du mystère et de la géniale improvisation. L'ouvrage collectif réalisé sous la direction de Philippe Hamon permet de nuancer la réponse. Il fait émerger, d'une manière remarquablement synthétique, les principes structurants de la méthode zolienne, tels qu'ils se donnent à lire dans l'ensemble des dossiers préparatoires. Mais il encourage aussi à décrypter le métadiscours d'un écrivain qui mêle constamment l'interrogation à l'invention et ne cesse, à la faveur d'un « soliloque programmatif » extrêmement dense, de dialoguer avec lui-même, d'ancrer son propos dans les problématiques contemporaines, en somme de poser l'écriture comme un acte complexe, impossible à circonscrire dans les limites du prévisible.

L'ouvrage invite à confronter les avant-textes zoliens aux catégories de la rhétorique classique : inventio, dispositio, elocutio, memoria et actio. La création ne se réduisant pas à la succession rectiligne de séquences fixes et exclusives les unes des autres, ce cadrage est rendu opérationnel par la reconnaissance d'un enchevêtrement permanent des opérations. Une telle démarche permet de mesurer l'efficacité des protocoles activés par l'auteur dans la phase prérédactionnelle, enrichis de dossier préparatoire en dossier préparatoire et nourris par une série de consignes plus ou moins récurrentes. L'un des principaux mérites de cet essai est d'aboutir ainsi à la compréhension globale des mécanismes impliqués dans la genèse des romans naturalistes. Il parvient à mêler une approche macroscopique destinée à éclairer la totalité d'une œuvre et une étude microscopique tenant compte des infimes détails grâce auxquels l'écriture zolienne signe sa différence.

Dans un premier chapitre, Alain Pagès s'attarde sur les différents niveaux de l'inventio : la question initiale, d'abord - Nana envisagé comme le «poème des désirs du mâle » ou Au Bonheur des dames comme celui « de l'activité moderne »-, puis surtout la recherche documentaire, essentielle dans une vision du monde où la logique doit prévaloir sur l'imagination. Les avant-textes témoignent de ce que la collecte des informations débouche sur l'obsession du classement et induit fréquemment le recours à des experts (Pol Lefèvre pour La Bête humaine, Alfred Roll pour Germinal, etc.) ou à des amis et correspondants avisés (Paul Alexis, Henry Céard). Alain Pagès envisage aussi la mise en scène du dilemme et la crainte lancinante de mal faire, telle qu'elle est trahie par les structures de l'alternative ou de l'épanorthose. 